\title{
Cardinality of sets of closed functional classes in weak 3 -valued logics
}

\author{
Nikolay N. PRELOVSKIY
}

\begin{abstract}
This paper proves that sets of closed functional classes in 3-valued logics of Bochvar $B_{3}$ and Hallden $H_{3}$ contains a continuum of different closed classes. It is also proven that both of these logics contain a closed functional class which has no basis.

Keywords: Bochvar's logic, Hallden's logic, closed class, continuum, cardinality
\end{abstract}

The research on cardinality of closed sets of functions in different logics was started by E. Post. Thus, in [10] he proved that classical logic only contains an enumerable set of different closed functional classes. In 1959 Yu.I. Yanov and A.A. Muchnik [3] for the first time showed that for every $k \geq 3$ the k-valued Post's logic $P_{k}$ contains a closed class which has no basis, and also contains a continual set of different closed functional classes. M.F. Ratsa in [4] and [5] showed that 3-valued logic of Heyting $G_{3}$ contains a continual set of different functional classes which have bases and a continual set of classes which have no bases. Consequently, cardinalities of sets of closed functional classes in different logics were researched in the fundamental monograph [9] by D. Lau which deals with functional algebras on finite sets.

A.S. Karpenko in [2] suggested a hypothesis that the set of closed classes in Bochvar's 3-valued logic $B_{3}$ has the power of continuum (truth-tables, defining basic functions of $B_{3}$, will be formulated below). As a justification of this hypothesis the author uses the condition (see [9, pp. 221-222]) for a class to contain just an enumerable set of closed functional classes. A.S. Karpenko found out that logic $B_{3}$ does not satisfy this criterion. Nevertheless, this condition is a 
sufficient but not necessary one. Despite this argument, we cannot conclude that the set of closed classes in $B_{3}$ is continual.

Thus, the question on cardinality of the set of closed classes in $B_{3}$ remained open until now. It was also unknown, whether there are functional classes in $B_{3}$ (id est logics weaker, than $B_{3}$ itself) which contain continual sets of closed classes. In this paper the author gives positive answers to these questions, and the answer to the latter may also be viewed as an answer to the former of them. In particular, as 3-valued Hallden's logic $H_{3}$ which was first studied in [8] contains (as shown below) a continual set of different closed classes, and as $H_{3}$ is precomplete in $B_{3}$ (this fact was proven by V.K. Finn in [6]), the set of closed classes in $B_{3}$ is continual.

Let us formulate a series of corresponding theorems and prove them. For this purpose we shall use the slightly modified strategy of Yu.I. Yanov and A.A. Muchnik.

Below we shall use basic functions of $B_{3}$, defined by the following truth-tables:

\begin{tabular}{|c|c|c|c|c|c|c|c|c|c|c|c|}
\hline$\cap$ & 1 & $\frac{1}{2}$ & 0 & $\cup$ & 1 & $\frac{1}{2}$ & 0 & $\sim$ & $x$ & $\vdash$ & $x$ \\
\hline 1 & 1 & $\frac{1}{2}$ & 0 & 1 & 1 & $\frac{1}{2}$ & 1 & 0 & 1 & 1 & 1 \\
\hline$\frac{1}{2}$ & $\frac{1}{2}$ & $\frac{1}{2}$ & $\frac{1}{2}$ & $\frac{1}{2}$ & $\frac{1}{2}$ & $\frac{1}{2}$ & $\frac{1}{2}$ & $\frac{1}{2}$ & $\frac{1}{2}$ & 0 & $\frac{1}{2}$ \\
\hline 0 & 0 & 1 & 0 & 0 & 1 & $\frac{1}{2}$ & 0 & 1 & 0 & 0 & 0 \\
\hline
\end{tabular}

TheOREM 1. The set of closed classes in $B_{3}$ contains a class, which has no basis.

Proof. Let us consider a sequence

$$
S=f_{0}, f_{1}\left(x_{1}\right), f_{2}\left(x_{1}, x_{2}\right), \ldots
$$

of functions $f_{i}\left(x_{1}, \ldots, x_{i}\right)$ of 3 -valued Post logic $P_{3}$ for $i \in\{0,1,2, \ldots\}$, satisfying the following conditions:

$$
\begin{gathered}
f_{0} \equiv 0 \\
f_{i}\left(x_{1}, \ldots, x_{i}\right)= \begin{cases}1 & \text { if } x_{1}=\ldots=x_{i}=\frac{1}{2} \\
0 & \text { otherwise }\end{cases}
\end{gathered}
$$

First of all, it is necessary to demonstrate that all the functions $f_{i}\left(x_{1}, \ldots, x_{i}\right)$ are in $B_{3}$. For this purpose it is sufficient to observe 
that the constant 0 is in $B_{3}$ and may be expressed with, for example, a formula $\vdash x \cap \sim \vdash x$, and to express the rest of the functions $f_{i}\left(x_{1}, \ldots, x_{i}\right)$ for every $i>0$ with formulas:

$$
\sim \vdash x_{1} \cap \sim \vdash \sim x_{1} \cap \ldots \cap \sim \vdash x_{i} \cap \sim \vdash \sim x_{i} .
$$

It is worth mentioning that the formula $\sim \vdash x \cap \sim \vdash \sim x$ represents the Rosser-Turquette operator ( $J$-operator) for the value $\frac{1}{2}$ in $B_{3}{ }^{1}$. So, the formula, expressing functions $f_{i}\left(x_{1}, \ldots, x_{i}\right)$, may be simplified with the use of $J$-operator for the value $\frac{1}{2}$ just as follows:

$$
J_{\frac{1}{2}}\left(x_{1}\right) \cap \ldots \cap J_{\frac{1}{2}}\left(x_{i}\right) .
$$

Let $\mathfrak{M}(S)$ be a class generated by the set of functions

$$
\left\{f_{0}, f_{1}\left(x_{1}\right), f_{2}\left(x_{1}, x_{2}\right), \ldots\right\} \subset B_{3}
$$

by renaming variables without identifying them. This class is a closed one. Let us also assume that $\mathfrak{M}(S)$ has a basis. In this case, there is a function $f^{\prime}$ that is obtained from function $f_{n_{0}}\left(x_{1}, \ldots, x_{n_{0}}\right)$ through renaming variables for which the number $n_{0}$ is minimal. Then we have two cases:

1. The basis contains at least one more function $f^{\prime \prime}$ corresponding to a function $f_{n_{1}}\left(x_{1}, \ldots, x_{n_{1}}\right)$ with $n_{1}>n_{0}$. As $f_{n_{0}}\left(x_{1}, \ldots, x_{n_{0}}\right)$ may be obtained from $f_{n_{1}}\left(x_{1}, \ldots, x_{n_{1}}\right)$ by identifying some of the variables $x_{1}, \ldots, x_{n_{1}}$, the function $f^{\prime}$ may be expressed through $f^{\prime \prime}$, and this contradicts to the definition of a basis.

2. The basis consists of a single function $f^{\prime}$. In this case no other function $f_{n}$ for $n>n_{0}$ can be expressed with $f^{\prime}$, as $f_{n_{0}}\left(\ldots, f_{n_{0}}, \ldots\right) \equiv$ 0 , that leads to a contradiction again.

THEOREM 2. There is a closed class with an enumerable basis in $B_{3}$.

Proof. To prove the theorem we shall consider a sequence

$$
S=f_{2}\left(x_{1}, x_{2}\right), f_{3}\left(x_{1}, x_{2}, x_{3}\right), \ldots
$$

\footnotetext{
${ }^{1}$ RosserTurquette operators $J_{1}(x)=\vdash x, J_{\frac{1}{2}}(x)=\sim \vdash x \cap \sim \vdash \sim x$ and $J_{0}(x)=\vdash \sim x$ for $B_{3}$ were for the first time constructed by V.K. Finn in [7].
} 
of functions $f_{i}\left(x_{1}, \ldots, x_{i}\right)$ in 3-valued logic of Post $P_{3}$ for $i \in$ $\{2,3, \ldots\}$, which satisfy the following conditions:

$$
f_{i}\left(x_{1}, \ldots, x_{i}\right)= \begin{cases}1 & \text { for } x_{1}=\ldots=x_{j-1}=x_{j+1}=\ldots=x_{i}=\frac{1}{2} \\ & x_{j}=1(1 \leq j \leq i) \\ 0 & \text { otherwise }\end{cases}
$$

Let us show that for every $i$ these functions can be defined using the basic functions of $B_{3}$. With this purpose for every $x_{j}(1 \leq j \leq i)$ and every $i$ we shall consider the following formulae of $B_{3}$ :

$F_{j}=\vdash x_{j} \cap\left(\sim \vdash x_{1} \cap \sim \vdash \sim x_{1}\right) \cap \ldots \cap\left(\sim \vdash x_{j-1} \cap \sim \vdash \sim x_{j-1}\right) \cap(\sim \vdash$ $\left.x_{j+1} \cap \sim \vdash \sim x_{j+1}\right) \cap \ldots \cap\left(\sim \vdash x_{i} \cap \sim \vdash \sim x_{i}\right)$.

Then let $F$ be the internal disjunction of formulae $F_{j}$ :

$$
F=\bigcup_{1}^{i} F_{j}
$$

For every fixed $i$, formulae $F$ define functions $f_{i}\left(x_{1}, \ldots, x_{i}\right)$ from $B_{3}$. Thus, for example, for $i=2$, there are only two formulae $F_{j}$, id est: $F_{1}=\vdash x_{1} \cap\left(\sim \vdash x_{2} \cap \sim \vdash \sim x_{2}\right)$ and $F_{2}=\vdash x_{2} \cap\left(\sim \vdash x_{1} \cap \sim \vdash \sim\right.$ $\left.x_{1}\right)$. Then $F=F_{1} \cup F_{2}$ is expressed in the following manner:

$\left(\vdash x_{1} \cap\left(\sim \vdash x_{2} \cap \sim \vdash \sim x_{2}\right)\right) \cup\left(\vdash x_{2} \cap\left(\sim \vdash x_{1} \cap \sim \vdash \sim x_{1}\right)\right)$.

It is easy to verify that the function $f_{2}\left(x_{1}, x_{2}\right) \in B_{3}$ corresponding to this formula returns the value 1 only on two tuples $\left\langle 1, \frac{1}{2}\right\rangle$ and $\left\langle\frac{1}{2}, 1\right\rangle$ of truth-values of variables $x_{1}$ and $x_{2}$. On all other tuples of truth-values this function returns the value 0 .

Thus, it is proven that for every $i$ functions $f_{i}\left(x_{1}, \ldots, x_{i}\right)$ are in $B_{3}$.

Notation of formulae $F_{j}$ and $F$ may be simplified essentially if we use $J$-operators for the truth-values 1 and $\frac{1}{2}$ :

$$
F_{j}^{\prime}=J_{1}\left(x_{j}\right) \cap J_{\frac{1}{2}}\left(x_{1}\right) \cap \ldots \cap J_{\frac{1}{2}}\left(x_{j-1}\right) \cap J_{\frac{1}{2}}\left(x_{j+1}\right) \cap \ldots \cap J_{\frac{1}{2}}\left(x_{i}\right) .
$$

Formula $F$, in this case, should be rewritten as the internal disjunction of all of the $F_{j}^{\prime}$ :

$$
F^{\prime}=\bigcup_{1}^{i} F_{j}^{\prime}
$$


Let $\mathfrak{M}(S)$ be a closed class generated by the system of functions $\left\{f_{2}\left(x_{1}, x_{2}\right), f_{3}\left(x_{1}, x_{2}, x_{3}\right), \ldots\right\}$. We shall prove that this system is a basis for $\mathfrak{M}(S)$. It is sufficient to show that none of the functions $f_{m}\left(x_{1}, \ldots, x_{m}\right)$ in this class can be expressed only with functions

$$
\left\{f_{2}\left(x_{1}, x_{2}\right), f_{3}\left(x_{1}, x_{2}, x_{3}\right), \ldots\right\} \backslash\left\{f_{m}\left(x_{1}, \ldots, x_{m}\right)\right\},
$$

id est there is no representation:

$$
f_{m}\left(x_{1}, \ldots, x_{m}\right)=\mathfrak{A}\left[f_{2}, \ldots, f_{m-1}, f_{m+1}, \ldots\right] .
$$

The formula $\mathfrak{A}\left[f_{2}, \ldots, f_{m-1}, f_{m+1}, \ldots\right]$ may be rewritten as:

$$
\begin{gathered}
\mathfrak{A}\left[f_{2}, \ldots, f_{m-1}, f_{m+1}, \ldots\right]= \\
=f_{r}\left(\mathfrak{B}_{1}\left[f_{2}, \ldots, f_{m-1}, f_{m+1}, \ldots\right], \ldots, \mathfrak{B}_{r}\left[f_{2}, \ldots, f_{m-1}, f_{m+1}, \ldots\right]\right) .
\end{gathered}
$$

Using the first equation, we have:

$$
\begin{gathered}
f_{m}\left(x_{1}, \ldots, x_{m}\right)= \\
=f_{r}\left(\mathfrak{B}_{1}\left[f_{2}, \ldots, f_{m-1}, f_{m+1}, \ldots\right], \ldots, \mathfrak{B}_{r}\left[f_{2}, \ldots, f_{m-1}, f_{m+1}, \ldots\right]\right) .
\end{gathered}
$$

Let us observe three possible cases:

1. At least two of the formulae among

$$
\mathfrak{B}_{1}\left[f_{2}, \ldots, f_{m-1}, f_{m+1}, \ldots\right], \ldots, \mathfrak{B}_{r}\left[f_{2}, \ldots, f_{m-1}, f_{m+1}, \ldots\right],
$$

where $r \geq 2$, do not coincide with symbols of variables. In this case, for every m-tuple $\left\langle\alpha_{1}, \ldots, \alpha_{m}\right\rangle$ of truth-values of variables $x_{1}, \ldots, x_{m}$, there are values 1 or 0 on corresponding argument places of the function

$$
f_{r}\left(\mathfrak{B}_{1}\left[f_{2}, \ldots, f_{m-1}, f_{m+1}, \ldots\right], \ldots, \mathfrak{B}_{r}\left[f_{2}, \ldots, f_{m-1}, f_{m+1}, \ldots\right]\right),
$$

and this function, according to its definition, will be equivalent to 0 . That is a contradiction to the hypothesis that the function $f_{m}\left(x_{1}, \ldots, x_{m}\right)$ may be expressed only with functions from

$$
\left\{f_{2}\left(x_{1}, x_{2}\right), f_{3}\left(x_{1}, x_{2}, x_{3}\right), \ldots\right\} \backslash\left\{f_{m}\left(x_{1}, \ldots, x_{m}\right)\right\},
$$


as no function in the set

$$
\left\{f_{2}\left(x_{1}, x_{2}\right), f_{3}\left(x_{1}, x_{2}, x_{3}\right), \ldots\right\}
$$

is equivalent to 0 .

2. Only one formula $\mathfrak{B}_{s}$ among

$$
\mathfrak{B}_{1}\left[f_{2}, \ldots, f_{m-1}, f_{m+1}, \ldots\right], \ldots, \mathfrak{B}_{r}\left[f_{2}, \ldots, f_{m-1}, f_{m+1}, \ldots\right]
$$

does not coincide with a symbol of variable. In this case, functions corresponding to the rest of the formulae in this list are equivalent to variables, and, as $r \geq 2$, there is at least one formula $\mathfrak{B}_{p} \equiv x_{q}$. Let us consider an $m$-tuple $\left\langle\alpha_{1}, \ldots, \alpha_{m}\right\rangle$ of truth-values for variables $x_{1}, \ldots, x_{m}$ such that $\alpha_{1}=\ldots=\alpha_{q-1}=\alpha_{q+1}=\ldots=\alpha_{m}=\frac{1}{2}$, and $\alpha_{q}=1$. On this ordered set of truth-values the function corresponding to the formula $\mathfrak{B}_{s}$ returns the value 1 or 0 . Then on the $m$-tuple $\left\langle\alpha_{1}, \ldots, \alpha_{m}\right\rangle$ of truth-values for variables $x_{1}, \ldots, x_{m}$ in the function

$$
f_{r}\left(\mathfrak{B}_{1}\left[f_{2}, \ldots, f_{m-1}, f_{m+1}, \ldots\right], \ldots, \mathfrak{B}_{r}\left[f_{2}, \ldots, f_{m-1}, f_{m+1}, \ldots\right]\right)
$$

there are at least two argument places having truth-values which do not coincide with $\frac{1}{2}$. Therefore, the right part of the equation is equal to 0 , and its left part must, according to definition of the function $f_{m}\left(x_{1}, \ldots, x_{m}\right)$, be equal to 1 which is impossible.

3. All of the formulae among

$$
\mathfrak{B}_{1}\left[f_{2}, \ldots, f_{m-1}, f_{m+1}, \ldots\right], \ldots, \mathfrak{B}_{r}\left[f_{2}, \ldots, f_{m-1}, f_{m+1}, \ldots\right]
$$

are equivalent to symbols of variables. Then $r>m$, and there are at least two entries of some variable $x_{p}$ in the formula expressing the function $f_{m}\left(x_{1}, \ldots, x_{m}\right)$. Considering the ordered $m$ tuple $\left\langle\alpha_{1}, \ldots, \alpha_{m}\right\rangle$ of truth-values for variables $x_{1}, \ldots, x_{m}$ such that $\alpha_{1}=\ldots=\alpha_{p-1}=\alpha_{p+1}=\ldots=\alpha_{m}=\frac{1}{2}$ and $\alpha_{p}=1$, we find out again that the right part of the corresponding equation is equal to 0 , and its left part is equal to 1 which is impossible.

All three cases lead to contradiction. Therefore, none of the functions $f_{m}\left(x_{1}, \ldots, x_{m}\right)$ where $m \geq 2$ can be represented as a formula using only functions from

$$
\left\{f_{2}\left(x_{1}, x_{2}\right), f_{3}\left(x_{1}, x_{2}, x_{3}\right), \ldots\right\} \backslash\left\{f_{m}\left(x_{1}, \ldots, x_{m}\right)\right\} .
$$


This theorem allows to prove one of the main results of this paper, that the set of closed classes in $B_{3}$ has the cardinality of continuum. The method for proving this result is the same with the strategy used by Yu.I. Yanov and A.A Muchnik, to prove continuality of the set of closed classes of functions in $k$-valued logics of Post $P_{k}$, for all $k \geq 3$.

THEOREM 3. The class of functions of $B_{3}$ contains a continuum of different closed sets.

Proof. The upper bound for cardinality of the set of closed classes in $B_{3}$ coincides with cardinality of the set of all subsets of functions in $B_{3}$. As the set of functions in $B_{3}$ is enumerably infinite, the set of all subsets of this set has the cardinality of continuum.

To obtain the lower bound for cardinality of the set of closed classes in $B_{3}$ it is enough to consider the closed class $\mathfrak{M}(S)$ constructed in the previous theorem. This class has a basis

$$
\left\{f_{2}\left(x_{1}, x_{2}\right), f_{3}\left(x_{1}, x_{2}, x_{3}\right), \ldots\right\} .
$$

For every sequence $S^{\prime}=s_{1}, s_{2}, \ldots$ of natural numbers, where $2 \leq$ $s_{1}<s_{2}<\ldots$, let us consider a closed class $\mathfrak{M}\left(S^{\prime}\right)$ which has a following set of functions as its basis:

$$
\left\{f_{s_{1}}\left(x_{1}, \ldots, x_{s_{1}}\right), f_{s_{2}}\left(x_{1}, \ldots, x_{s_{2}}\right), \ldots\right\} .
$$

It is obvious that

$$
\mathfrak{M}\left(s_{1}, s_{2}, \ldots\right) \neq \mathfrak{M}\left(s_{1}^{\prime}, s_{2}^{\prime}, \ldots\right),
$$

if $\left\{s_{1}, s_{2}, \ldots\right\} \neq\left\{s_{1}^{\prime}, s_{2}^{\prime}, \ldots\right\}$.

Consequently, the set of closed classes $\left\{\mathfrak{M}\left(S^{\prime}\right)\right\}$ in the set of closed classes of $B_{3}$ is continual.

A question arises, whether existence of a set

$$
\left\{f_{2}\left(x_{1}, x_{2}\right), f_{3}\left(x_{1}, x_{2}, x_{3}\right), \ldots\right\}
$$

of functions defined in the previous manner in a functional class of some 3 -valued logic is necessary for this logic to contain a continuum 
of different closed classes. In general, the answer to this question is 'wrong', as the above-formulated definition of the sequence $S$ of functions $f_{i}\left(x_{1}, \ldots, x_{i}\right)(i \geq 2)$ is, in a certain sense, too strong, because it requires possibility to use at least two $J$-operators, as we can do in $B_{3}$. But prerequisites of this definition may be weakened essentially, so that we shall be able to prove one of the key theorems of this paper about continuality of the set of closed classes in 3valued logic of Hallden $\mathrm{H}_{3}$.

The basic connectives of logic $H_{3}$ are those in the set $\left\{\sim, J_{\frac{1}{2}}, \cap, \cup\right\}$ (for example, see [1, p. 57]).

THEOREM 4. The set of closed functional classes in $H_{3}$ contains a class, which has no basis.

Proof. The sequence of functions

$$
S=f_{0}, f_{1}\left(x_{1}\right), f_{2}\left(x_{1}, x_{2}\right), \ldots
$$

is defined, using Rosser-Turquette operator $J_{\frac{1}{2}}(x)$, just as it was done in Theorem 1. The rest of the proof is completely analogous to the proof of Theorem 1 .

THEOREM 5. The class of functions in $H_{3}$ contains a closed class, which has an enumerable basis.

Proof. To prove the theorem, consider a sequence

$$
S=f_{2}\left(x_{1}, x_{2}\right), f_{3}\left(x_{1}, x_{2}, x_{3}\right), \ldots
$$

of functions $f_{i}\left(x_{1}, \ldots, x_{i}\right)$ of 3 -valued logic of Post $P_{3}$, for $i \in$ $\{2,3, \ldots\}$, satisfying the following definition:

$$
f_{i}\left(x_{1}, \ldots, x_{i}\right)= \begin{cases}1 & \text { if } x_{1}=\ldots=x_{j-1}=x_{j+1}=\ldots=x_{i}=\frac{1}{2} \\ & x_{j} \in\{1,0\}(1 \leq j \leq i) \\ 0 & \text { otherwise }\end{cases}
$$

Let us show that such functions may be defined using the basic functions of $H_{3}$, for each $i$. With this purpose we need to consider, for every $x_{j}(1 \leq j \leq i)$ and every $i$, the following formulae of $H_{3}$ : 


$$
F_{j}=\sim J_{\frac{1}{2}}\left(x_{j}\right) \cap J_{\frac{1}{2}}\left(x_{1}\right) \cap \ldots \cap J_{\frac{1}{2}}\left(x_{j-1}\right) \cap J_{\frac{1}{2}}\left(x_{j+1}\right) \cap \ldots \cap J_{\frac{1}{2}}\left(x_{i}\right) .
$$

Then, for every $i$, let $F$ be the internal disjunction of all of the formulae $F_{j}$ :

$$
F=\bigcup_{1}^{i} F_{j} .
$$

The rest of the proof is analogous to the corresponding proof for $B_{3}$.

THEOREM 6. There is a continuum of different closed functional classes among functions of $\mathrm{H}_{3}$.

Proof. The proof of this theorem is identical with the corresponding proof for $B_{3}$.

It is worth mentioning that proofs of theorems 4-6 do not depend on proofs of theorems $1-3$, and, as $H_{3}$ is precomplete in $B_{3}$, the former of them may be viewed as independent proofs of corresponding facts for $B_{3}$.

After proving these theorems one can suppose that enjoying the property of having a continuum of different closed functional classes for various multi-valued logics is rather a normal phenomenon, than a strange deviation, even for very weak multi-valued functional systems like $H_{3}$. If this hypothesis is true, it may be viewed as a new philosophical argument enforcing the thesis about qualitative difference between multi-valued logics and classical bivalent logic.

\section{References}

[1] Karpenko A. S., Development of Multivalued Logic. M.: URSS, 2010 (in Russian).

[2] Karpenko A. S., Continuality of 3-Valued Logics: Problems and Hypotheses, Logical Investigations 16:127-133, 2010. (in Russian)

[3] Muchnik A. A., Yanov Yu. I., On Existence of $k$-valued Closed Classes, Which Have no Finite Bases, Proceedings of Academy of Sciences USSR 127(1):44-46, 1959 (in Russian).

[4] Ratsa M. F., On Functional Completeness of Propositional Intuitionistic Logic, Problems of Cybernetics 39:107-150, 1982 (in Russian). 
[5] Ratsa M. F., Iterative Chain Classes of Pseudo-boolean Functions. Kishinev, 1990 (in Russian).

[6] Finn V. K., On Criterion of Functional Completeness for $B_{3}, R e$ searches in Formal Languages and Non-classical Logics, M.: Nauka, 1974, pp. 194-199 (in Russian).

[7] Finn V. K., Axiomatization of Some 3-valued Propositional Calculi and Their Algebras, Philosophy in Modern World. Philosophy and Logic, M.: Nauka, 1974, pp. 398-438 (in Russian).

[8] Hallden S., The Logic of Nonsense, Uppsala Universitets Arsskrift, 1949.

[9] LAu D., Function Algebras on Finite Sets, Springer-Verlag, BerlinHeidelberg-New York, 2006.

[10] Post E. L., The two-valued iterative systems of mathematical logic, Annals of Mathematical Studies 5, Princeton University Press, 1941. 